Edvard Govekar, Andrej Jeromen, Alexander Kuznetsov, Gideon Levy, Makoto Fujishima, Study of an annular laser beam based axially-fed powder cladding process,

CIRP Annals,

Volume 67, Issue 1,

2018,

Pages 241-244,

ISSN 0007-8506.

(C) 2018. This manuscript version is made available under the CC-BY-NC-ND 4.0 license http://creativecommons.org/licenses/by-nc-nd/4.0/

Link to published article:

http://www.sciencedirect.com/science/article/pii/S0007850618301069

DOI: https://doi.org/10.1016/j.cirp.2018.04.082 


\title{
Study of an annular laser beam based axially-fed powder cladding process
}

\author{
Edvard Govekar (1)a, Andrej Jeromen a, Alexander Kuznetsov a, Gideon Levy(1) ${ }^{\mathrm{b}}$, Makoto Fujishima (3)c \\ a University of Ljubljana, Faculty of Mechanical Engineering, Ljubljana, Slovenia \\ b TTA Technology, Switzerland \\ c DMG Mori , CO., LTD., 2-35-16 Meieki, Nakamura-ku, Nagoya City Aichu 450-0002, Japan
}

An annular laser beam based powder cladding head, which enables an axial powder feeding and variation of the laser beam intensity distribution (LBID) on the workpiece surface is presented. The influence of typical LBIDs, including Ring, Tophat(-), Tophat(+), and Gaussian-like, on a cladding process has been characterized based on the process and melt pool visualization, powder catchment efficiency, clad layer geometry, and porosity. The results showed that the most stable process without plasma formation but with low dilution and porosity of the clad layer can be achieved within the range from a Ring to a Tophat(-) LBID. Additionally, axial powder feeding results in a high powder catchment efficiency above $80 \%$.

Additive manufacturing, Laser, Powder

\section{Introduction}

In laser metal powder cladding, the geometrical and metallurgical properties of a clad material are governed by complex laser beam - melt pool - powder stream interactions, which besides the process parameters are also influenced by the geometrical relations of the laser beam caustic and powder stream. The later are defined by the design of the cladding head which includes a powder nozzle, that is used to form and inject the powder stream into the melt pool created on a workpiece surface by the laser beam. Depending on the application, in commercially available cladding heads, usually a Gaussian laser beam is used, and powder is fed laterally by a single nozzle or coaxially by several discrete or a continuous nozzles [1]. The application of a Gaussian laser beam with laterally or coaxially fed powder can cause overheating at the centre of the clad, and insufficient heating at its edge. This can lead to undesirable, deep, non-uniform, and even asymmetrical dilution with a weak bond at the edge of the clad due to the higher powder density, laser beam attenuation, and higher cooling rates at the edge compared to the center of the clad [2]. Additionally, the side and coaxial powder feeding, results in a relatively low powder catchment efficiency of less than $50 \%$ [3]. In order to increase the powder catchment efficiency and to generate clads having the desired properties, the laser beam intensity and the powder density distribution should be adjusted so that a uniform heat input can be achieved over the whole width of the clad spot. For this purpose an annular laser beam cladding head, which enables axial powder feeding has been proposed [4-6].

In the paper, a novel annular laser beam (ALB) powder cladding head is presented, including a laser beam shaping unit, which, in addition to axial powder feeding, makes it possible to vary the laser beam intensity distribution (LBID) from Ring to Gaussianlike. From the presented experimental results, the advantage of axial powder feeding and the important influence of LBID on powder cladding process stability, powder catchment efficiency, and the related characteristics of the clad layer are evident. It is considered that an LBID within the range from a Ring to a Tophat is the most appropriate in the case of axially-fed fed metal powder cladding.

\section{Annular laser beam - axial fed powder cladding head}

The novel annular laser beam (ALB) - axial fed powder cladding head is presented schematically in Fig. 1a. It consists of a laser beam shaping unit, a guiding mirror, a laser beam focusing unit, an axial powder delivery tube and nozzle, and a coaxial shielding gas nozzle. A collimated laser beam passes through the laser beam shaping unit to be shaped into an ALB. By means of the mirror the ALB is then guided coaxially to the axis of the powder delivery tube into the focusing unit, where it is focused on the workpiece surface. In Fig. 1b some photos showing laser beam shaping and focusing units with the axial powder nozzle and the coaxial shielding gas nozzle are shown. The laser beam shaping unit can be used to shape various ALB caustics by varying the position of lens L1, which is located between the axicons A1 and A2. In Fig. 1c an example of an IR camera based measured ALB caustic with related LBID vs. the workpiece surface laser beam focal plane standoff position $l_{\mathrm{fw}}$ is shown. The related convergence $\theta=17^{\circ}$ and wedge angle $\gamma=1.5^{\circ}$ of the caustic were defined based on the detection of the $1 / \mathrm{e}^{2}$ intensity boundaries of the LBI distribution IR images on thin metal foil, acquired at different workpiece standoff positions $l_{\mathrm{fw}}$. It can be seen that by varying the position $l_{\mathrm{fw}}$, the LBID and the related laser beam outer diameter $d_{0}$ can be changed. Similarly the LBID can be modified by varying the position $l_{1}$ of lens L1 and of the related ALB caustic itself. By varying both positions, $l_{\mathrm{fw}}$ and $l_{1}$, various LBIDs can be generated, from Ring to Gaussian-like, at various outer diameters $d_{0}$. An example of a Tophat LBID with a variable outer diameter $d_{0}$ is shown in Fig. 1d. 
a)

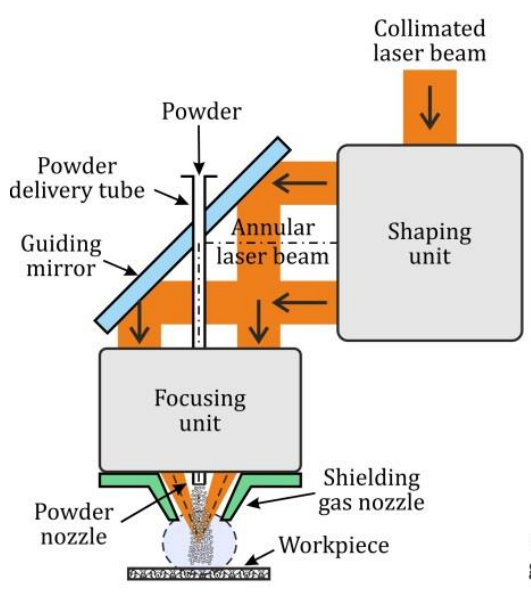

b)
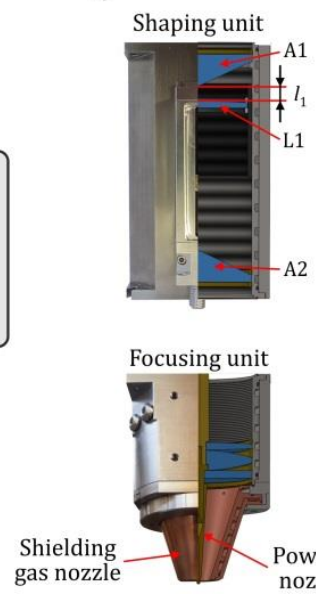

Focusing unit

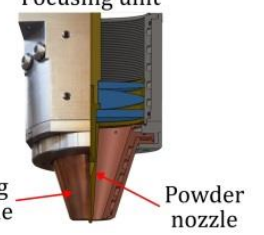

c)

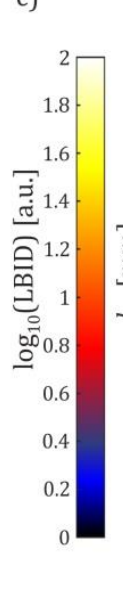

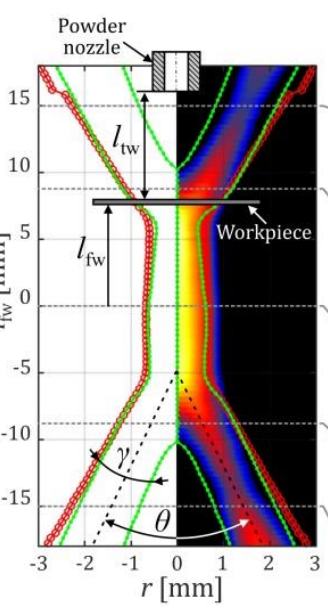

d)

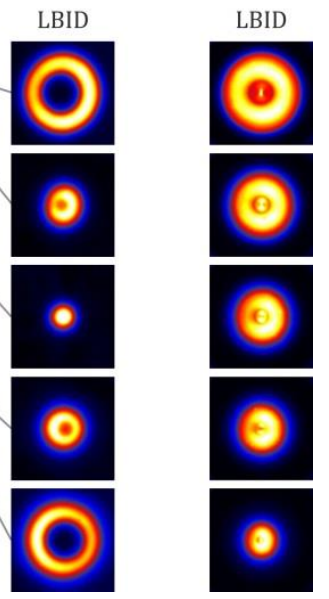

Figure 1. a) General arrangement of the ALB, axially-fed powder cladding head, b) Photos of laser beam shaping and focusing units with shielding gas and axial powder nozzles, c) Annular laser beam caustic and related LBIDs $I_{\mathrm{lb}}, \mathrm{d}$ ) Example of generated LBID obtained by variation of $l_{1}$ and $l_{\mathrm{fw}}$.

Application of the ALB enables axial feeding of the powder into the centre of the laser beam circular spot and the related melt pool generated on the workpiece surface. In the case of such feeding less laser beam attenuation, and higher powder catchment efficiency can be expected. In our case, a simple nozzle with an inner diameter $d_{\mathrm{p}}=1.0 \mathrm{~mm}$ was used for this purpose. In order to characterize the powder stream at the exit from the powder nozzle, a visualization setup consisting of a vertical illumination line diode laser of wavelength $660 \mathrm{~nm}$ and a CMOS camera was used. The camera was positioned perpendicularly to the laser illumination plane through the axis of the powder stream. In Fig. 2a an image of the powder stream obtained by superposition of $5 \times 10^{4}$ images of powder particles at the exit from the powder nozzle is shown. Based on the powder particle image analysis, powder particle density distributions along the radius $r$ were estimated at various values of the powder nozzle standoff distance $l_{\mathrm{tw}}$, as shown in Fig. 2a. By calculating the related cumulative distribution $F(r)$ of the powder particles, the related powder stream radius and diameter $d \%$ respectively, within which a certain \% of the powder stream is contained, can be defined depending on the powder nozzle standoff distance $l_{\mathrm{tw}}$, as shown in Fig. 2 b. It can be seen that at a distance $l_{\mathrm{tw}}=8.0 \mathrm{~mm}$, the $90 \%$ powder stream diameter $d_{90}$ is $5.6 \mathrm{~mm}$.
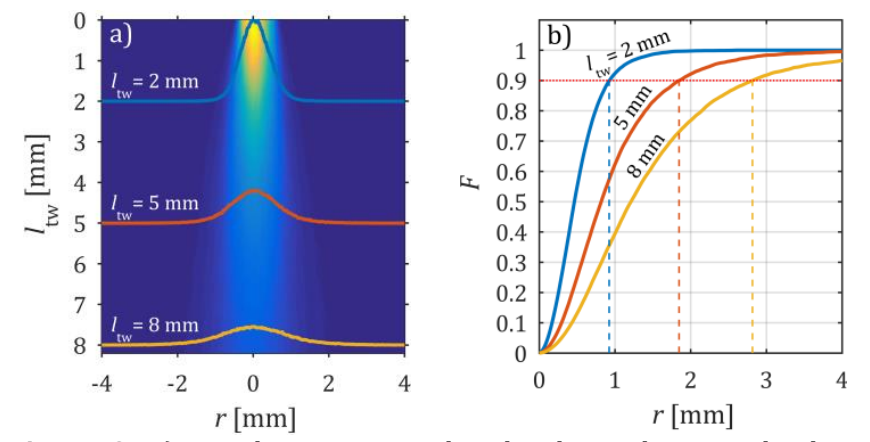

Figure 2. a) Powder stream with related powder particle density distribution vs. $r$ at various positons $l_{\mathrm{tw}}, \mathrm{b}$ ) related Cumulative powder density function $F$ vs. powder stream radius $r$ at various $l_{\mathrm{tw}}$.

\section{Experimental}

In powder cladding experiments, the upper part $\left(l_{\mathrm{fw}}>0 \mathrm{~mm}\right)$ of the ALB caustic was used in order to ensure shorter powder nozzle standoff distances $l_{\text {tw }}$ and to avoid passing the powder through the high LBID region. Fig. 3a presents IR images of the four generated characteristic Ring, Tophat(-), Tophat(+), and
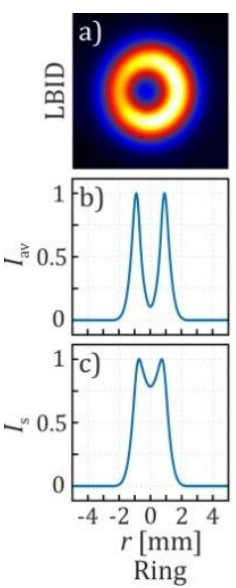
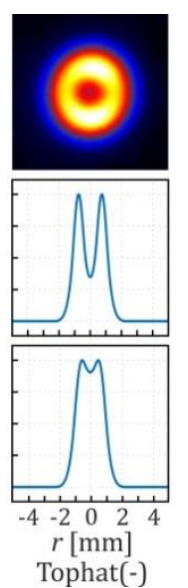
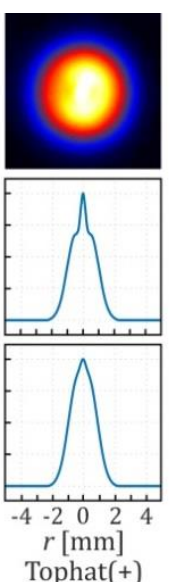
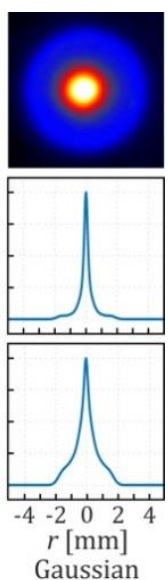

Figure 3. a) IR camera based images of LBID, b) related average intensity profiles $I_{\text {av }}$, and c) scanning intensity profiles $I_{\mathrm{s}}$.

Gaussian like LBIDs, with a four sigma outer diameter $d_{\mathrm{lb}}=3.0$ $\mathrm{mm}$, which were used to investigate the influence of LBID on the axially fed powder cladding process. Using $1 / \mathrm{e}^{2}$ criteria, the thickness of the Ring was $d_{\mathrm{lb}} / 3$. Additionally, the average laser beam intensity profiles $I_{\mathrm{av}}$ and the related scanning laser beam intensity profiles $I_{\mathrm{s}}$ are shown in Fig. 3b and 3c. The latter are obtained by integrating the IR camera based two dimensional LBID in the feed direction and describing the energy input distribution across the irradiated path.

Values of process parameters used in the powder cladding experiments are shown in Table 1. As the energy source, a CW diode laser with a wavelength range of 900-1100 nm was used. Using SS316L powder with a particle size span from 53 to $125 \mu \mathrm{m}$, cladding was performed on a SS304 workpiece with dimensions $(60 \times 25 \times 10) \mathrm{mm}$. Argon was used as a powder carrier and shielding gas. To feed the powder, a roller based powder feeder was used.

Table 1. Powder cladding process parameters.

\begin{tabular}{lc} 
Parameter & Value \\
\hline Laser beam power $-P[\mathrm{~kW}]$ & $1.5,2.0$ \\
Feed velocity $-v_{\mathrm{f}}[\mathrm{mm} / \mathrm{min}]$ & $5.0,10.0$ \\
Laser beam outer diameter $-d_{o}[\mathrm{~mm}]$ & 3.0 \\
Powder mass flow $-\Phi_{\mathrm{m}}[\mathrm{g} / \mathrm{min}]$ & $8.5,17.0$ \\
Powder carrier gas volume flow $-\Phi_{\mathrm{vp}}[1 / \mathrm{min}]$ & 0.5 \\
Shielding gas volume flow $-\Phi_{\mathrm{vs}}[\mathrm{l} / \mathrm{min}]$ & 5.0 \\
Standoff distance $-l_{\mathrm{tw}}[\mathrm{mm}]$ & 8.0
\end{tabular}




\section{Influence of LBID on the cladding process}

The influence of LBID (Fig. 3) on the cladding process when performed by axially fed powder into the centre of the LBID, as well as the related clad layer properties, are now presented. The process was analysed based on process visualisation, geometrical characterisation of the clad layer, powder catchment efficiency, and the porosity of the single and multiple layers.

\subsection{Melt pool visualisation and process stability}

For the process visualisation and characterisation of the influence of LBID on the cladding process and the melt pool, a CMOS camera with 633 fps was used. In Fig. 4a characteristic images of the melt pool at different LBIDs are shown. The black dots indicate incoming, axially fed, non-melted powder. From the

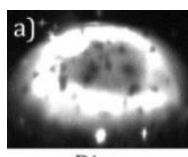

Ring

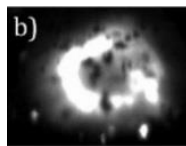

$t=0.663 \mathrm{~s}$

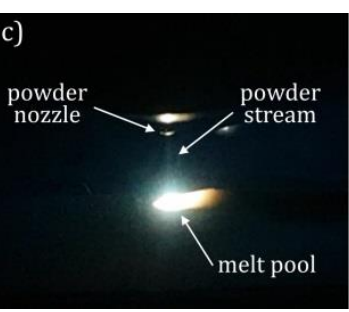

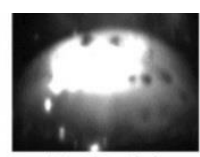

Tophat $(+)$

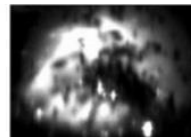

$t=0.672 \mathrm{~s}$

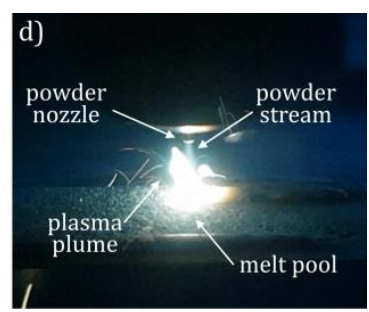

Figure 4. a) Melt pool at various LBID, b) Sequence of melt pool images in the case of Tophat(-) LBID, c) Stable cladding process, d) Unstable cladding process with the presence of a plasma plume.

Tophat(-) related melt pool image sequence shown in Fig. 4b, a non-stationary i.e. highly pulsating powder stream can be seen due to the applied roller based powder feeding unit. It can also be seen that at certain process parameters $\left(P, v_{\mathrm{f}}, \Phi_{\mathrm{m}}\right)$ with Ring and Tophat(-) LBID, the powder cladding process runs in a stable manner (Fig. 4c), whereas at the Tophat $(+)$ and Gaussian-like LBID, a strong undesirable plasma plume is generated above the clad layer surface, as shown in Fig. 4d. The reason for the observed plasma plume is the high laser beam intensity at the centre of distribution into which the metal powder is delivered, causing powder particle evaporation.

\subsection{Clad layer geometrical properties}

At different generated LBID, the properties of the clad layer geometry including the layer width $w$, the height $h$ and the maximum penetration depth $h_{\text {md }}$ were analysed in dependence on the main process parameters $P, v_{\mathrm{f}}$, and $\Phi_{\mathrm{m}}$. It was shown that the LBID itself does not qualitatively influence the relations between $P, \quad \mathrm{f}_{,}, \Phi_{\mathrm{m}}$ and $w, h, h_{\mathrm{md}}$. As stated in the literature [7] and graphically presented in Fig. 5, with increasing laser beam power $P$, a constant to slightly increasing clad layer width $w$ and an increasing height $h$ and clad layer maximum penetration depth $h_{\text {md }}$ can be observed. With an increase in the feed velocity $v_{\text {f }}$, the related properties $w, h$ and $h_{\text {md }}$ decrease in size. An increase in the powder mass flow $\Phi_{\mathrm{m}}$ results in a constant to a slight increase
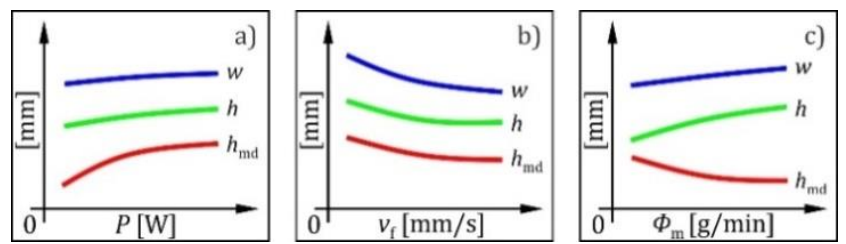

Figure 5. Qualitative relations between the process parameters $P, v_{\mathrm{f}}, \Phi_{\mathrm{m}}$ and the related clad layer geometrical characteristics $w, h$, and $h_{\mathrm{md}}$.

in the clad layer width $w$, an increase in the clad layer height $h$, and a decrease in the maximum penetration depth $h_{\mathrm{md}}$.

With the aim of quantitatively characterizing the influence of LBID on clad layer properties in the case of axially fed powder, the resulting cross-sections of the clads were prepared and geometrically compared. An example of cross-section photos of the clads generated with different LBIDs are shown in Fig. 6, where the first row corresponds to $P=1.5 \mathrm{~kW}$ and the second row to $P=2.0 \mathrm{~kW}$ at a feed velocity $v_{\mathrm{f}}=10.0 \mathrm{~mm} / \mathrm{s}$ and a powder mass flow $\Phi_{\mathrm{m}}=8.5 \mathrm{~g} / \mathrm{min}$. It first can be seen that the LBID

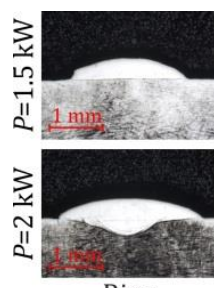

Ring

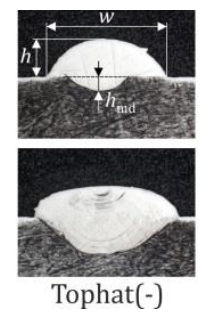

Tophat(-)

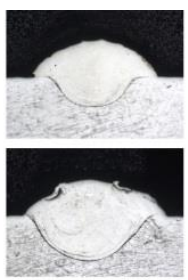

Tophat $(+)$

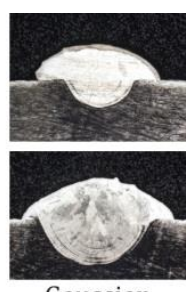

Gaussian
Figure 6. Clad cross sections vs. LBI distribution at $P=1.5$ and $2.0 \mathrm{~kW}$.

significantly influences the geometrical properties of the clad. For Ring LBID, a small constant or slightly U-shaped dilution with a less pronounced maximum penetration depth $h_{\mathrm{md}}$ at the centre of the clad is characteristic. When the LBID is varied towards Gaussian-like, the depth of the U-shaped dilution increases. In the case of LBID with a high intensity at the centre, a lack of dilution and insufficient melting can be observed along the edges, due to the low LBI and higher heat conduction into the workpiece at the clad edges. In Fig. 7, the geometrical characteristics of clads are plotted against the LBID at $P=2.0 \mathrm{~kW}$. It can be seen that the clad width $w$ remains almost constant, whereas the height $h$ increases as the LBID is changed from Ring towards Gaussian-like. As can be seen from the images of the clad cross-sections, the most sensitive to LBID variation is the clad dilution shape and the related maximal penetration which increases when the LBID is varied from RING towards Gaussian-like. Additionally, the dependence of $h_{\mathrm{md}}$ on $v_{\mathrm{f}}$ and $\Phi_{\mathrm{m}}$ is presented at the bottom of the diagram in Fig. 8. The results of the experiments also showed that, with an increase in the laser beam power $P$, the observed effects of LBID variation become less pronounced.

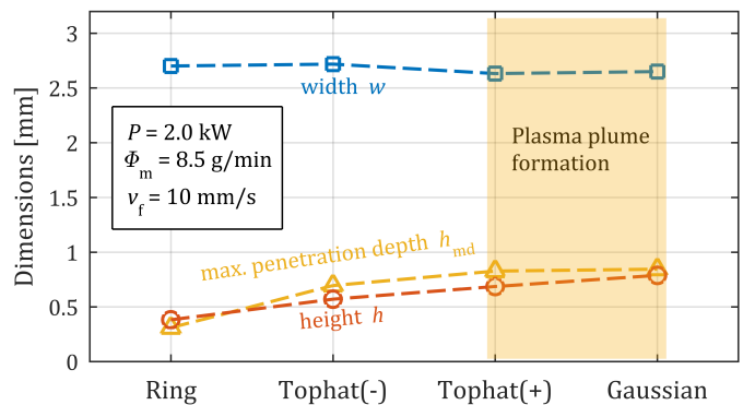

Figure 7. Clad geometrical characteristics $w, h$, and $h_{\text {md }}$ vs. LBID. 


\subsection{Metal powder catchment efficiency}

In order to characterise the powder catchment efficiency $\eta_{\mathrm{p}}$ achieved with the axial feed powder cladding process, the ratio between the clad layer mass $m_{c}$ and the estimated delivered powder mass $m_{\mathrm{d}}$ was calculated. The clad mass $m_{\mathrm{c}}$ was measured by means of a scale with a measurement resolution $1 \mathrm{mg}$ and uncertainty $2 \mathrm{mg}$. The delivered powder mass $m_{d}$ was estimated based on a long-run experimentally defined average powder mass flow generated by the powder feeder unit. The results of powder catchment efficiency including $\pm \sigma$ error bars, achieved at a laser beam power of $P=2.0 \mathrm{~kW}$, with various LBIDs and process parameters, are shown in Fig. 8. It can firstly be seen that, in the axially fed powder cladding process, a very high powder catchment efficiency $\eta_{\mathrm{p}}$ above $80 \%$ can be achieved. It can also be seen that an increase in the feed velocity $v_{\mathrm{f}}$ causes a decrease in

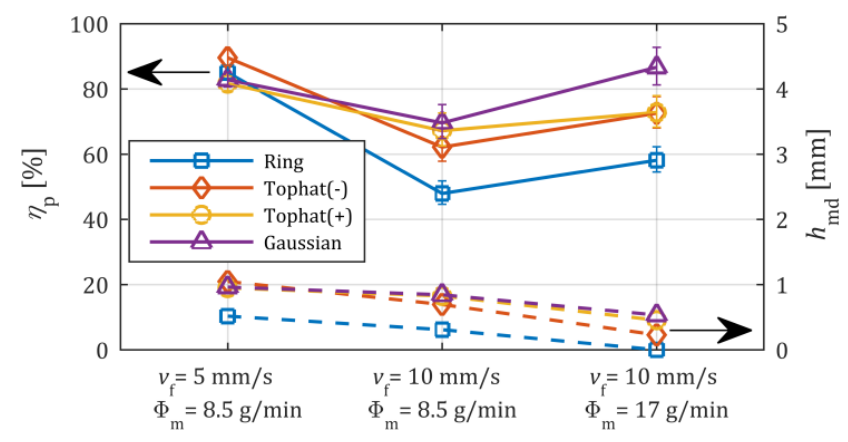

Figure 8. Powder catchment efficiency $\eta_{\mathrm{p}}$ and maximum penetration depth $h_{\mathrm{md}}$ vs. $v_{\mathrm{f} \text {, and }} \Phi_{\mathrm{m}}$ at various LBIDs.

the powder catchment efficiency $\eta_{\mathrm{p}}$, whereas an increase in the powder mass flow $\Phi_{\mathrm{m}}$ results in an increase in the powder catchment efficiency $\eta_{\mathrm{p}}$. From the point of view of the LBID, the powder catchment efficiency $\eta_{\mathrm{p}}$ increases when the LBID is varied from Ring towards Gaussian-like. The relations presented in Fig. 8 are linearly dependent on the laser beam power $P$, and as the latter is increased the differences in powder catchment efficiency $\eta_{\mathrm{p}}$, achieved by different LBID, decrease. However, the application of the centrally concentrated Tophat(+) and Gaussian-like LBIDs are limited by plasma plume formation, which makes the Ringlike LBIDs more appropriate.

\subsection{Clad layer porosity}

An important property of the clad is also its porosity, which was characterised by porosity area fraction $\varepsilon$ of the clad cross section [8]. In our case the pores larger than $10 \mu \mathrm{m}$ observed on images of 4-layered clad cross sections were considered. Cladding was performed at laser power $P=1.5$ and $2.0 \mathrm{~kW}$ with feed velocity $\nu_{\mathrm{f}}=10.0 \mathrm{~mm} / \mathrm{s}$ and a powder mass flow $\Phi_{\mathrm{m}}=8.5 \mathrm{~g} / \mathrm{min}$. The
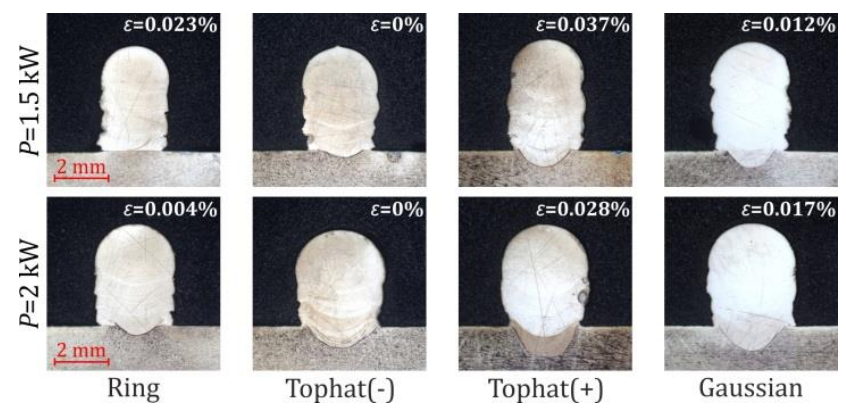

Tophat(-) related values of porosity $\varepsilon$ are indicated in the top right corner of the cross-section photos in Fig. 9. It can be seen that in general the process of axial fed powder cladding, regardless of the LBID, results in a low value of porosity $\varepsilon<0.037 \%$ comparable with coaxial powder cladding [9].

\section{Conclusions}

In the paper, a novel powder cladding head with a designed annular laser beam shaping unit was presented. The head besides an axial powder feeding into the center of the generated melt pool enables variation of the LBID on the workpiece surface.

In order to investigate the influence of the LBID on the axiallyfed powder cladding process, four different LBIDs, varying from Ring to Gaussian-like, with an outer laser beam diameter $d_{0}=3.0 \mathrm{~mm}$, were generated and applied to the cladding of SS $316 \mathrm{~L}$ powder on a SS 304 workpiece surface. It was shown that with axial powder feeding, applying suitable process parameters, a high powder catchment efficiency $\eta_{\mathrm{p}}$ above $80 \%$ can be achieved, which increases when the LBID is varied from Ring to Gaussian-like. In all cases of LBIDs, a low porosity was also observed. However, using LBID with high intensity at the centre of the distribution, i.e. Tophat $(+)$ and Gaussian-like, can result in the undesirable formation of a plasma plume due to overheating of the axially fed powder and to the lack of a proper metallurgical bond at the edge of the clad. In the other hand, in the case of Ring and Tophat(-) LBIDs the achieved high powder catchment efficiency and dilution with a good metallurgical bond at the clad edge can be further improved by increasing the laser beam power and the related intensity at the clad edge, without the presence of a plasma plume.

Based on the experimental results it can be concluded that axial powder feeding is very beneficial from the point of view of high powder catchment efficiency. In a combination with a proper LBID a low dilution with good bonding at the clad edges, and low porosity of clads can be achieved. Depending on the process parameters the most appropriate LBID is to be found in the range from Ring to Tophat. In our case, Tophat(-) LBID was selected. However, in order to define an optimal LBID, a multi-parametric optimisation should be performed.

\section{Acknowledgments}

The authors acknowledge the financial support received from DMG Mori Co., Ltd. and the Slovenian Research Agency (research core funding No. P2 0241).

\section{References}

[1] Lamikiz, A., Tabernero, I., Ukar, E., Martinez, S., Lopez de Lacalle, l., N., 2011, Current Designs of Coaxial Nozzels for Laser Cladding, Recent Patents on Mechanical Engineering, 4: 29-36.

[2] de Lange, D.F., Hofman, J.T., Meijer, J., 2005, Influence of intensity distribution on the meltpool and clad shape for laser cladding, Proc. Third Int. WLT - Conference on Lasers in Manufacturing, 323-327.

[3] Dias da Silva, M., Partes, K., Seefeld, T., Volllertsen, F., 2012, Comparison of coaxial and off-axis nozzle configurations in one step process laser cladding on aluminum substrate, J. Mater. Process. Technol., 212: 2514- 2519.

[4] Liu, S., Kong, F., Shi, S., Kovacevic R., 2014, Study of a hollow laser beam for cladding, Int. J. Adv. Manuf. Tech., 73:147-159.

[5] Kuznetsov, A., Jeromen, A., Levy, G., Fujishima, M., Govekar, E., 2016, Annular laser beam cladding process feasibility study, Phys. Proc., 83: 647-656.

[6] Fujishima, M., Govekar, E., Levy, G.N., 2017, Head for additive processing, processing machine, and processing method, Patent W02017115406.

[7] Sun, Y., Hao, M., 2012, Statistical analysis and optimization of process parameters in Ti6Al4V laser cladding using Nd:YAG laser, Opt. Lasers Eng., 50: 985-995.

[8] Majumdar, J., D., Pinkerton, A., Liu, Z., Manna, I., Li, L., 2005, Microstructure characterisation and process optimization of laser assisted rapid fabrication of 316L stainless steel, Appl. Surf. Sci., 247: 320-327.

[9] Fujishima, M., Oda, Y., Ashida, R., Takezawa, K., Kondo, M., 2017, Study on factors for pores and cladding shape in the deposition processes of Inconel 625 by the directed energy deposition (DED) method, CIRP J. Manuf. Sci. Technol., 19: 200204.

Figure 9. Four layered clad cross sections vs. LBID at $P=1.5$ and $2.0 \mathrm{~kW}$, with related porosity $\varepsilon$. 